\title{
Evaluation of the effects of power-frequency magnetic fields on the electrical activity of cardiomyocytes differentiated from human induced pluripotent stem cells
}

\author{
Masayuki Takahashi'1 , Atsushi Saito', Yasuhiko Jimbo² and Satoshi Nakasono' ${ }^{1}$ \\ ${ }^{I}$ Environmental Science Research Laboratory, Central Research Institute of Electric Power Industry (CRIEPI), \\ 1646 Abiko, Abiko-shi, Chiba 270-1194, Japan \\ ${ }^{2}$ Graduate School of Engineering, The University of Tokyo, 7-3-1 Hongo, Bunkyo-ku, Tokyo 113-8656, Japan
}

(Received December 5, 2016; Accepted January 11, 2017)

\begin{abstract}
Although cardiac activity is known to differ between species in many respects, most evaluations of the cardiac effects of low-frequency electric and magnetic fields, which have a stimulant effect on electrically activated cells, have been performed in non-human experimental animals and cells, and the effects in humans have been assessed using theoretical models. In recent years, it has been verified that human cardiomyocytes differentiated from human induced pluripotent stem cells (hiPS-CM) are useful for evaluating human responses to various cardioactive compounds. In this study, we applied hiPS$\mathrm{CMs}$ for the first time to evaluate the human cardiac effects of power-frequency magnetic fields (MFs). After preparation of hiPS-CMs, we subjected a hiPS-CM monolayer formed on a multi-electrode array to short-term exposure to a $50 \mathrm{~Hz} \mathrm{MF}$ at $400 \mathrm{mT}$ with recording of the extracellular field potentials. The field potential duration of the hiPS-CMs did not differ significantly pre- and post-exposure, indicating that under these conditions, exposure to a $50 \mathrm{~Hz}$ MF at $400 \mathrm{mT}$ does not affect the electrical activity of hiPSCMs.
\end{abstract}

Key words: Power-frequency magnetic field, Cardiomyocyte, Human induced pluripotent stem cell, Extracellular field potential

\section{INTRODUCTION}

Exposure to time-varying and sufficiently strong electric and magnetic fields (EMFs) is known to have a stimulant or thermal effect on human tissues. Sensitivity to the two effects differs depending on the frequency of the EMF. EMFs $<100 \mathrm{kHz}$ have a predominantly stimulant effect. In the current international guidelines/standards for protecting the human body from low-frequency $(<100 \mathrm{kHz})$ EMF exposure (The Institute of Electrical and Electronics Engineers, 2002; International Commission on NonIonizing Radiation Protection, 2010), threshold values that stimulate neurons in the central and peripheral nervous systems are used as the basis for restrictions because these are the cells with the highest sensitivity to EMFs (Reilly, 1998, 2002).

Muscle cells are also electrically activated cells. In particular, continuous rhythmic depolarization and repolarization of cardiomyocytes $(\mathrm{CMs})$ is required for beat- ing of the heart. Cardiac hazards, such as the threshold value for ventricular fibrillation, have been evaluated by magnetic or electrical stimulation of experimental animals (Elmas et al., 2012; Fozzard and Schoenberg, 1972; Mouchawar et al., 1989), and data from animal studies have been applied to humans after adjusting for body size (Geddes et al., 1973). However, theoretical models are limited in their ability to predict human responses, as it is known that many aspects of fundamental cardiac activity, such as beating rate and electrocardiogram results (Boukens et al., 2014; Denning et al., 2016), differ between species. Although some human studies have been reported, the strength of EMF exposure was sufficiently low to be safe (Cook et al., 1992; Korpinen et al., 1993; Graham et al., 1994; Korpinen and Partanen, 1994), and limited experimental data are available for direct evaluation of human effects. In Environmental Health Criteria 238 (World Health Organization, 2007) published by the World Health Organization, it was concluded that hazard-

Correspondence: Masayuki Takahashi (E-mail: takamasa@criepi.denken.or.jp) 
ous cardiovascular effects associated with low-frequency EMFs are unlikely to occur at the exposure levels commonly encountered environmentally or occupationally; furthermore, the current international guidelines/standards based on nervous system sensitivity prevent cardiac hazard from electric stimulation, including that induced by exposure to low-frequency EMFs. However, because insufficient accumulation of knowledge regarding biological effects might reduce the reliability of risk assessment, and the effect on CMs is potentially life-threatening, it would be valuable to directly evaluate the responsiveness of human CMs to stimulant effects of low-frequency EMFs.

Human induced pluripotent stem (hiPS) cells, which are generated by reprogramming somatic cells using a particular set of transcription factors, have the ability to differentiate into multiple cell lineages, including CMs (Takahashi et al., 2007; Yu et al., 2007). CMs derived from hiPS cells (hiPS-CMs) are artificially generated source of human tissue, and their potential for use in medical and pharmacological applications has been intensively examined (Funakoshi et al., 2016; Kawamura et al., 2012; Khan et al., 2013; Sirenko et al., 2013). In the pharmacological field, hiPS-CMs have been confirmed to show appropriate responsiveness to known cardioactive compounds (Sirenko et al., 2013), and hiPS-CMs are expected to contribute to efficient screening of candidate drugs (Khan et al., 2013). HiPS-CMs are becoming a powerful tool to evaluate human responses. In this study, we applied hiPS-CMs for the first time to evaluate the biological effects of EMFs. After preparation of human CMs differentiated from hiPS cells, we subjected the hiPS-CMs to short-term exposure to a $50 \mathrm{~Hz}$ magnetic field (MF) at $400 \mathrm{mT}$ and evaluated the effects by comparing the pre- and post-exposure extracellular field potentials of the hiPS-CMs.

\section{MATERIALS AND METHODS}

\section{Cell culture}

The hiPS cell line (201B7) (Takahashi et al., 2007) was provided by the RIKEN BRC through the Project for Realization of Regenerative Medicine and the National Bio-Resource Project of the MEXT, Japan. The undifferentiated hiPS cells were maintained on a mitotically inactivated SNL76/7 feeder layer in Primate ES Cell Medium (ReproCELL, Kanagawa, Japan) supplemented with $10 \mathrm{ng} / \mathrm{mL}$ basic fibroblast growth factor (Wako, Tokyo, Japan). The culture medium was changed daily and the cells were passaged every 5 days.

\section{Embryoid body formation and cardiomyogenic differentiation}

The iPS cell colonies were detached with dissociation solution for human ES/iPS cells (ReproCELL). To induce formation of embryoid bodies (EBs), the colonies were transferred to plastic Petri dishes and cultured in $\alpha$ MEM (Gibco, Grand Island, NY, USA) supplemented with 20\% Knockout Serum Replacement (KSR; Gibco) and $0.1 \mathrm{mM} \beta$-mercaptoethanol (Gibco). Wnt-3a (R\&D Systems, Minneapolis, MN, USA) was added $(100 \mathrm{ng} / \mathrm{mL}$ ) to the medium from days 1 to 3 (Tran et al., 2009; Yazawa et al., 2011). On day 6, the EBs were plated into gelatin-coated $35-\mathrm{mm}$ culture dishes in aMEM supplemented with 5\% fetal bovine serum (FBS; Gibco), $0.1 \mathrm{mM} \beta$-mercaptoethanol, 100 units $/ \mathrm{mL}$ penicillin and $100 \mu \mathrm{g} / \mathrm{mL}$ streptomycin. Subsequently, $50 \%$ of the medium was replaced every 3 or 4 days. Beating EBs were mechanically isolated under a microscope (Eclipse TS100, Nikon, Tokyo, Japan), and trypsinized to prepare cell suspensions of cardiomyocytes.

\section{Immunofluorescence}

Cell suspensions of hiPS-CMs were reseeded on the gelatin-coated interior of a handmade polydimethylsiloxane (PDMS) O-ring (inner diameter: $8 \mathrm{~mm}$ ) adhered to a chamber slide. The cardiomyocyte monolayer that formed after more than a week of culture was fixed using 4\% paraformaldehyde for $30 \mathrm{~min}$ at $4{ }^{\circ} \mathrm{C}$ and permeabilized using $0.2 \%$ Triton $\mathrm{X}-100$ for $15 \mathrm{~min}$ at room temperature. Then the cells were blocked with 10\% FBS followed by incubation with primary antibodies, such as mouse IgG1 monoclonal anti- $\alpha$ actinin (1:400 dilution; Sigma-Aldrich, Bellefonte, PA, USA), mouse IgG1 monoclonal anti-MHC (1:100; Abcam, Cambridge, UK), mouse IgG2b monoclonal anti-Troponin T (1:100; Abcam), mouse IgG2a monoclonal anti-GATA4 (1:50; Santa Cruz Biotechnology, Santa Cruz, CA, USA), goat polyclonal antiNkx2.5 (1:50; Santa Cruz Biotechnology), and rabbit polyclonal anti-ANP (1:50; Santa Cruz Biotechnology). The secondary antibodies were all purchased from Molecular Probes (Eugene, OR, USA): Alexa Fluor 488-conjugated goat anti-mouse IgG1 (1:500) and chicken anti-mouse $\operatorname{IgG}(1: 500)$ and Alexa Fluor 568-conjugated goat anti-mouse IgG2a (1:500), donkey anti-goat $\operatorname{IgG}(1: 500)$, and goat anti-rabbit $\operatorname{IgG}$ $(1: 200)$. Stained samples were observed by fluorescent microscopy (Eclipse Ti-U, Nikon).

\section{Multi-electrode array (MEA) recording}

Extracellular field potential (FP) recordings of the 
Evaluations of the human cardiac effects of magnetic fields

hiPS-CMs were performed using the MEA system (MED64; Alpha MED Scientific, Osaka, Japan) and the accompanying software (Mobius QT). For drug loading tests, a hiPS-CMs monolayer was formed on an electrode array positioned in the center of the probe (MED-P515A; Alpha MED Scientific) using a PDMS O-ring as described above. The O-ring was removed several days later. ReproCardio2 Assay Medium (Repro CELL) was used as a recording medium. For testing of MF exposure, cardiomyocyte monolayers were formed independently on two areas of an electrode array in a probe (MED-P5003A). To provide higher exposure of hiPS$\mathrm{CMs}$ to induced electric fields, a probe with electrode areas far from the center was used. Data were analyzed using the Mobius QT software.

\section{Drug loading test}

After the medium on the MEA probe was exchanged for recording medium, the probe was incubated in the environmental chamber of the microscope at $37^{\circ} \mathrm{C}$ for $90 \mathrm{~min}$. Then, the FPs were recorded for $5 \mathrm{~min}$. Subsequently, a stock solution of the drug to be tested was added to the medium at $0.1 \%$ dilution and the FPs were recorded for $5 \mathrm{~min}$ following a 10 -min incubation. The drugs tested were E-4031, verapamil, and aspirin (all from Sigma-Aldrich).

\section{MF Exposure}

For MF exposure, we used a previously reported system (Nakasono et al., 2003), with different settings. The system comprised a pair of saddle-shaped coils and a window frame-shaped iron core (Fig. 1A), which can generate a vertical sinusoidal MF with a magnetic flux density of up to $400 \mathrm{mT}(\mathrm{rms})$ at $50 \mathrm{~Hz}$. The MF was measured using a Gaussmeter (Model 9550; F.W. Bell, Orlando, FL, USA) and determined to show $>95 \%$ uniformity. A sample stand was installed in the middle of the coils. The stand was fixed on the foundation independent of the coils to exclude vibration artifacts. An acrylic chamber (Fig. 1B) was also placed on the stand. The chamber has separate thin spaces on the top, bottom, and rear for circulating heated fluoric inactive solution (Fluorinert; $3 \mathrm{M}$, St. Paul, MN, USA), and the internal temperature was maintained at $37 \pm 0.5^{\circ} \mathrm{C}$. During the MF exposure tests, an iPS-CM monolayer on an MEA probe, which was placed in a connector with a cable, was positioned in the chamber. All of the metallic components of the connector, with the exception of the circuit board, cable plug, and cable connector, were replaced with acrylic substitutes. After one hour of acclimatization, the FPs of the iPS-CMs were recorded for
$3 \mathrm{~min}$. The cells were exposed sequentially to a $50 \mathrm{~Hz} \mathrm{MF}$ at 0 and $400 \mathrm{mT}$ for $9 \mathrm{sec}(3 \mathrm{sec}$ of 0 to $400 \mathrm{mT}, 3 \mathrm{sec}$ of $400 \mathrm{mT}$, and $3 \mathrm{sec}$ of 400 to $0 \mathrm{mT}$ ), followed by $3 \mathrm{~min}$ of recording of the iPS-CM signals. The 9-sec MF exposure was controlled by a function generator (WF1974; NF, Yokohama, Japan). Finally, the iPS-CMs were incubated

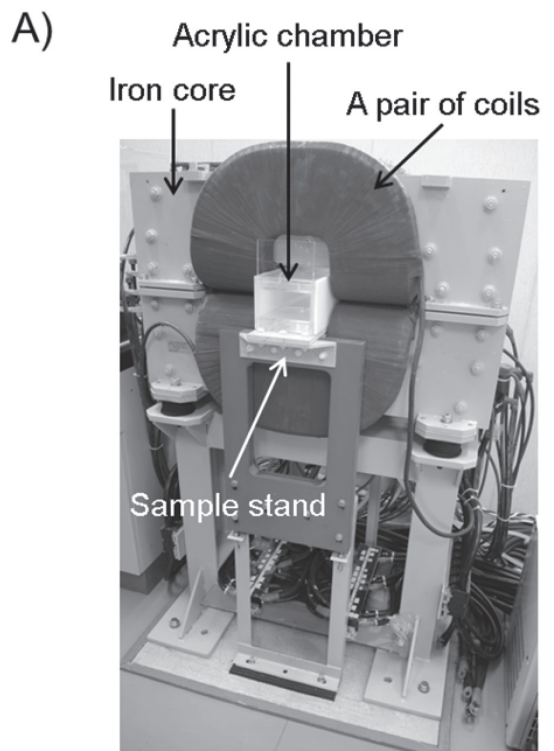

B)

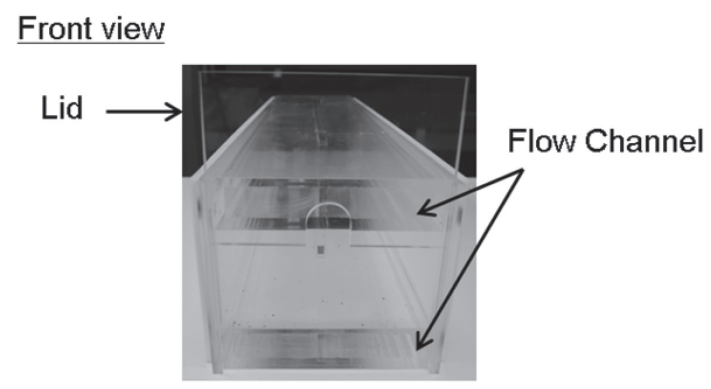

Side view

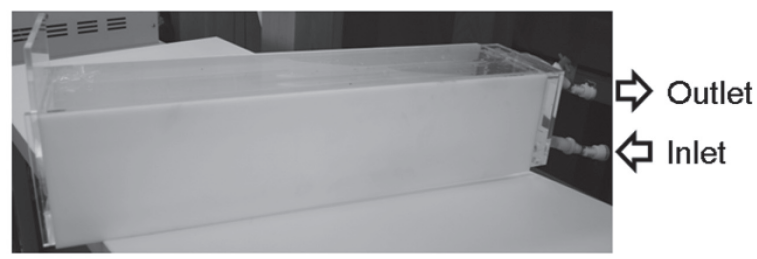

Fig. 1. Magnetic field (MF) exposure system. A: A pair of saddle-shaped coils and a window frame-shaped iron core. B: Acrylic chamber for temperature control. 
A)
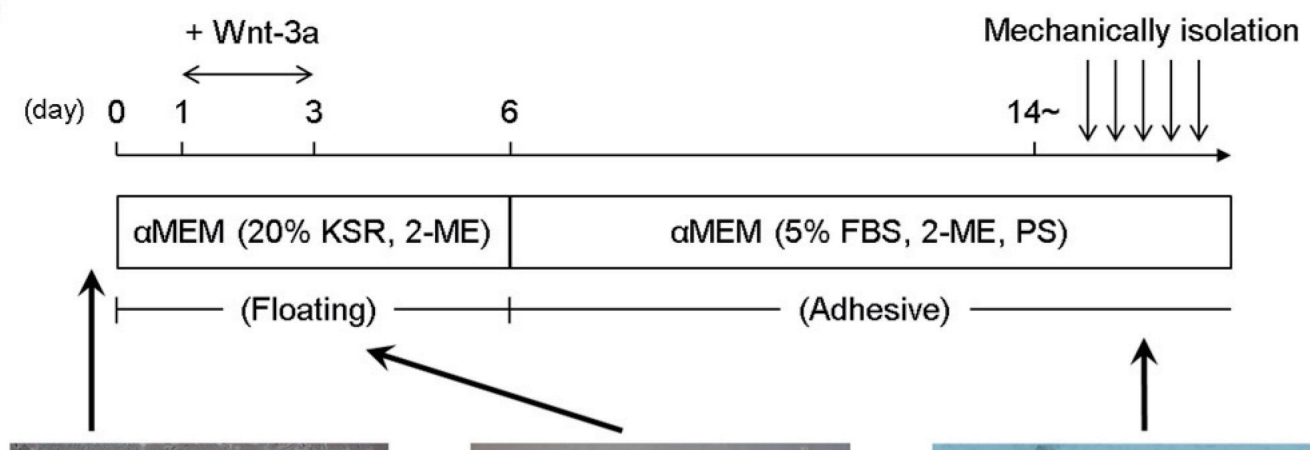

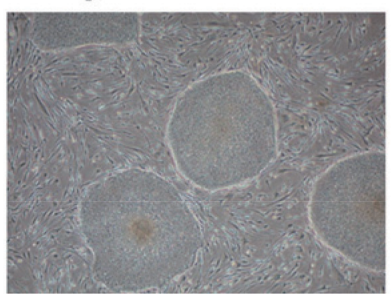

hiPS colonies

B)

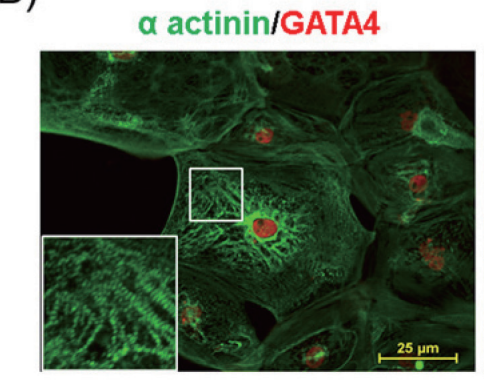

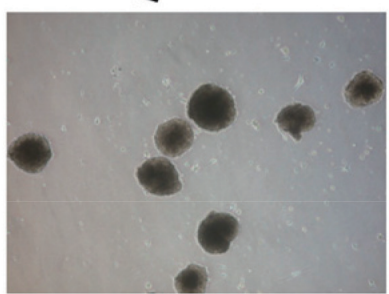

EBs

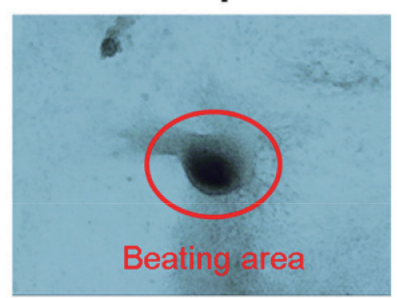

hiPS-CMs
Troponin TIANP

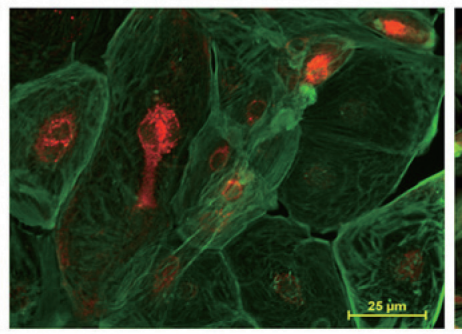

MHC/Nkx2.5

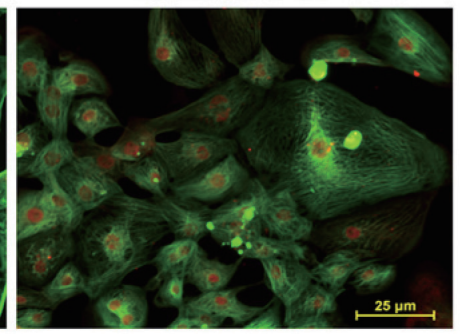

Fig. 2. Differentiation of human induced pluripotent stem (hiPS) cells into cardiomyocyes (CMs). A: Schematic of the differentiation protocol. After formation of embryoid bodies (EBs) from hiPS colonies, the EBs were attached to gelatin-coated culture dishes. During the cultivation period, beating CMs derived from hiPS cells (hiPS-CMs) were mechanically isolated under a microscope and used for further experiments. B: Immunofluorescence staining of hiPS-CMs. Fabricated hiPS-CM monolayers were stained with antibodies against the cardiac-specific proteins $\alpha$ actinin, GATA4, troponin T, ANP, MHC, and Nkx2.5.

with $200 \mathrm{nM}$ of E-4031 for 15 min followed by recording the FPs for 3 min (Fig. 4A). The magnetic flux density in the exposure space was confirmed using a Narda ELT-400 with $3 \mathrm{~cm}^{2}$ probe (Pfullingen, Germany).

\section{Statistical analysis}

Data are expressed as means \pm standard deviation (S.D.), and significant differences between groups were determined using the 2-sample Student's $t$-test. P-values $<0.05$ were considered statistically significant.

\section{RESULTS}

\section{Differentiation of human iPS cells to cardiomyocytes}

We induced differentiation of human iPS cells to cardiomyocytes using a general method for EB formation (Fig. 2A). Some of the EBs started beating spontaneously approximately 14 days after differentiation. Initially, to characterize the induced cells, we prepared a monolayer of beating EBs and performed immunofluorescence staining. The cells were positively stained with antibodies 
Evaluations of the human cardiac effects of magnetic fields

directed against the cardiac-specific transcription factors GATA4 and Nkx2.5 and the cardiac structural proteins $\alpha$ actinin, myosin heavy chain (MHC), and troponin $\mathrm{T}$ (Fig. 2B). Sarcomeric organization with well-organized cross-striation and atrial natriuretic peptide (ANP) in the secretory granules surrounding the nuclei were also observed. These results confirmed the formation of a hiPS-CMs monolayer.

\section{Pharmacological features of human iPS cell-derived cardiomyocytes}

We next evaluated the pharmacological features of the hiPS-CMs. Changes in the FPs of hiPS-CMs following drug loading were monitored using an MEA system. FP duration (FPD), which is the time interval from the depolarization spike to the peak of the repolarization phase, was measured (Fig. 3A). The FPD is known to correspond to the duration of the action potential, which reflects the QT interval in an electrocardiogram (Reppel et al., 2007; Halbach et al., 2003; Caspi et al., 2009). Figure 3B indicates that the $I_{\mathrm{kr}}$ channel blocker E-4031 prolonged the FPD in a concentration-dependent manner and the calcium channel blocker verapamil shortened the FPD concentration-dependently. The same changes of FPD were confirmed following each drug loading in two other independent experiments. Thus, the induced hiPS-CMs exhibited normal pharmacological sensitivity.

\section{Responses of human iPS cell-derived cardiomyocytes to $50 \mathrm{~Hz}$ magnetic fields}

To study the responsiveness of hiPS-CMs to powerfrequency MF, we exposed a hiPS-CM monolayer on an MEA probe to a $50 \mathrm{~Hz} \mathrm{MF}$ and simultaneously recorded the FPs of the cells pre- and post-exposure (Fig. 4A). Representative FPD data collected from a single sample exposed to a $50 \mathrm{~Hz}$ MF are shown in Fig. 4B. The FPDs of pre-exposed hiPS-CMs were not significantly altered by exposure to a $50 \mathrm{~Hz} \mathrm{MF}$ at $400 \mathrm{mT}$ for $3 \mathrm{sec}$ (Fig. 4C). The MF-exposed hiPS-CMs were confirmed to exhibit normal sensitivity to E-4031 (Fig. 4C). The same results were observed in two other independent experiments with different hiPS-CM samples. These results suggested that short-term exposure to a $50 \mathrm{~Hz} \mathrm{MF}$ at $400 \mathrm{mT}$ does not disrupt the electrical activity of cardiomyocytes.

\section{DISCUSSION}

Here, we evaluated the stimulant effect of power-frequency MF on the electrical activity of human CMs using CMs differentiated from hiPS cells. HiPS-CMs have recently attracted considerable attention, not only

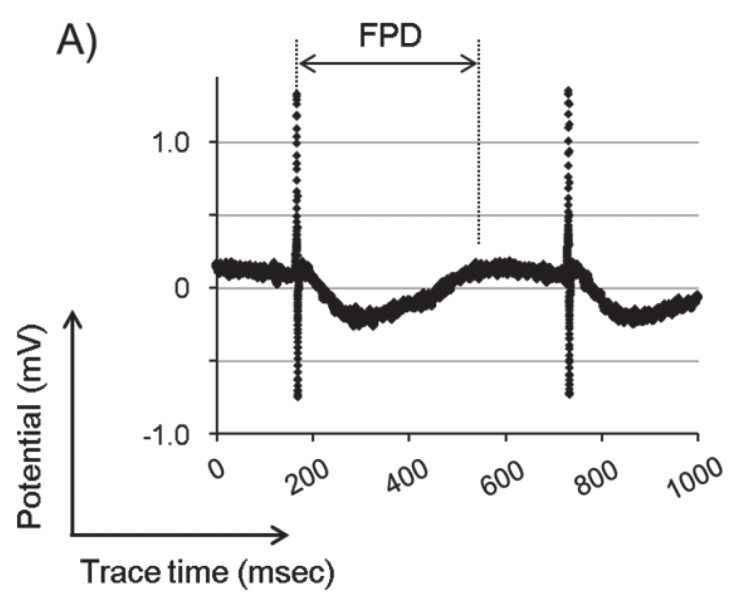

B)
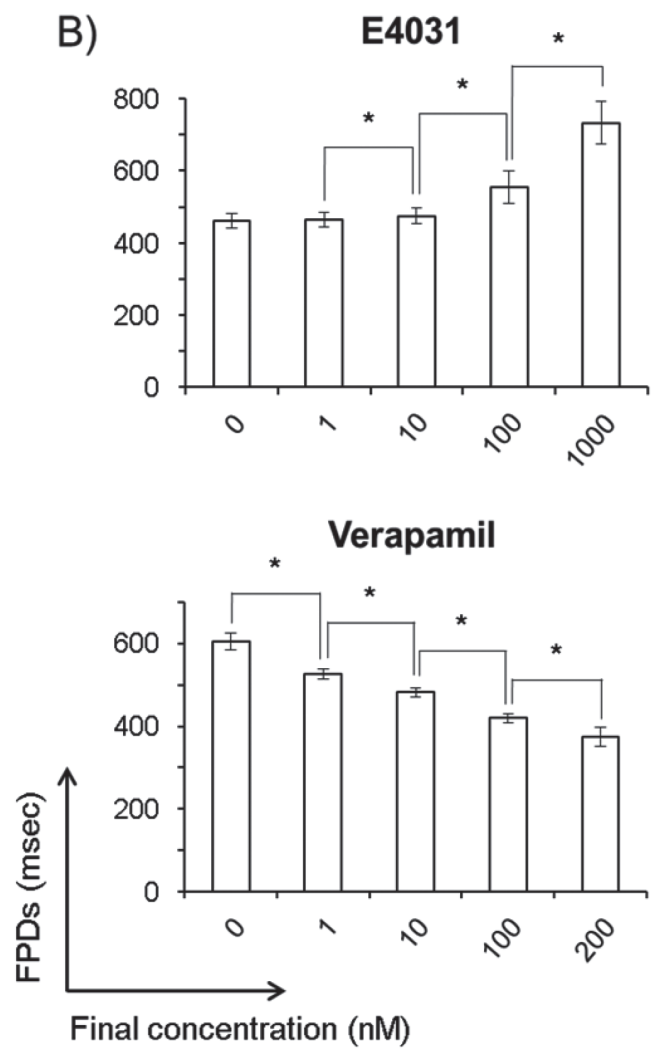

Fig. 3. Characterization of hiPS-CMs. A: Extracellular field potential (FP) of a hiPS-CMs monolayer recorded using a multi-electrode array (MEA) system. The time interval from the depolarization spike to the peak of the repolarization phase was used as the FP duration (FPD). B: Drug loading of the hiPS-CMs monolayer. After loading each concentration of a stock solution of each drug, the FP of the hiPS-CM monolayer was recorded and changes in the FPDs were analyzed. Each bar represents the mean and SD of all FPDs for each recording time. $* \mathrm{P}<0.01$. 
M. Takahashi et al.

A)
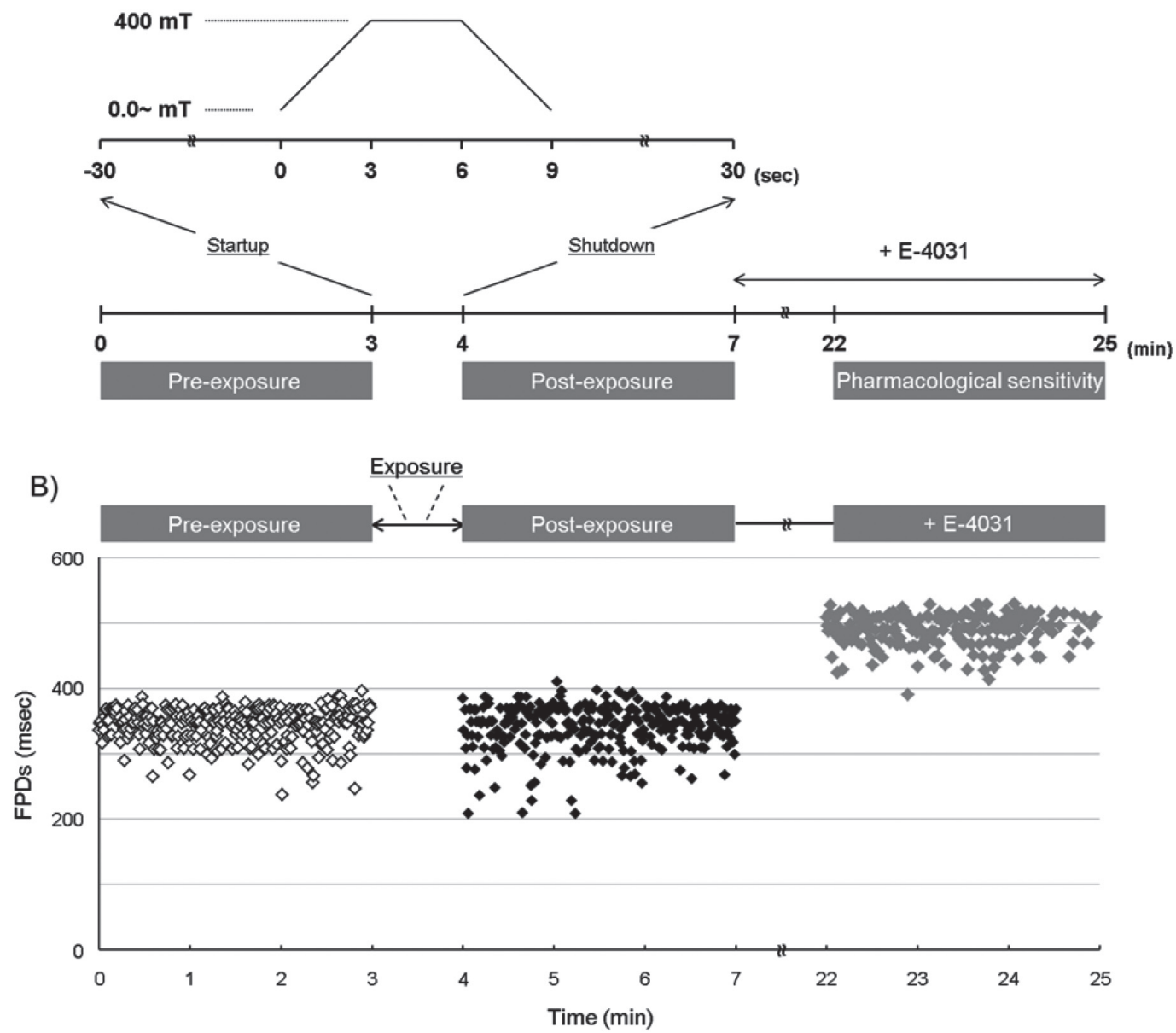

C)

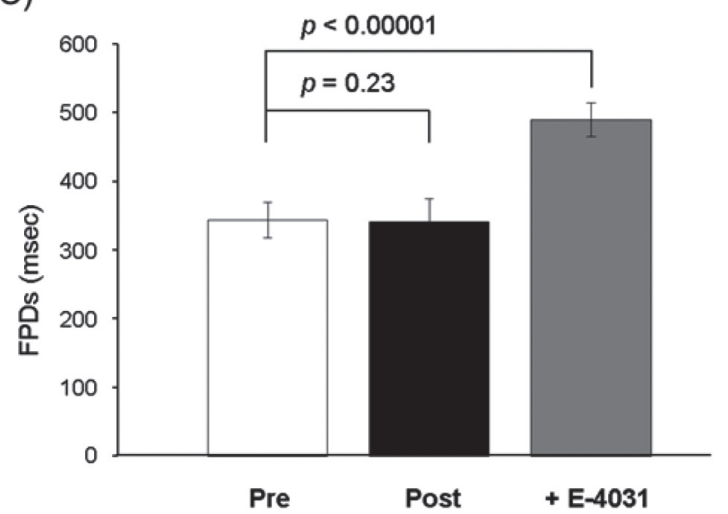

Fig. 4. Responses of hiPS-CMs to a $50 \mathrm{~Hz}$ magnetic field. A: Experimental design for magnetic field (MF) exposure and field potential (FP) recordings. A hiPS-CMs monolayer was exposed to a $50 \mathrm{~Hz} \mathrm{MF}$ at $400 \mathrm{mT}$ and the FPs were recorded pre- and post-exposure. After MF exposure, the pharmacological sensitivity of the sample was confirmed. B: FP duration (FPD) recorded from a sample. Although electrical noise was generated during initiation of the MF exposure system, the FPs were recorded continuously through each experiment. C: Comparison of FPDs pre-exposure, post-exposure, and following addition of E-4031. Each bar represents the mean and SD of all FPDs in each period recorded from a sample. 
Evaluations of the human cardiac effects of magnetic fields

for their potential medical uses for recovery of cardiac function, but also for their potential pharmaceutical uses. Cardiac activity is known to differ between humans and experimental animals. Beating rate and electrocardiogram results differ greatly between species, as do the molecular mechanisms of repolarization and expression of various genes and proteins (Boukens et al., 2014; Denning et al., 2016). These cause differences in each species' tolerance to chemical agents (Price et al., 2008), and might yield false-positive or -negative results when human toxicity is evaluated using experimental animals and cells. Tests using many kinds of known cardioactive compounds have shown that hiPS-CMs are useful for evaluation of pharmacological responsiveness in humans (Sirenko et al., 2013; Khan et al., 2013). Thus, assay systems using hiPS-CMs are likely to provide accurate and efficient screening of candidate drugs. HiPS-CMs also show potential as a powerful tool to evaluate biological effects in health risk assessment. In this study, we applied hiPS$\mathrm{CMs}$ to evaluate the effects of power-frequency MFs. To the best of our knowledge, this is the first report in which cells derived from iPS cells were used for assessment of human responses to non-ionizing radiation.

The effects of low-frequency EMFs on the electrical activity of CMs have previously been evaluated in vivo in dogs and rats and in vitro in mouse and rat CMs (Cornacchione et al., 2016; Wei et al., 2015). Despite the importance of gathering human-specific data, given interspecies differences in cardiac activity, few studies for risk assessment have been performed on human tissues. Some studies in which the strength of EMF exposure was sufficiently low to be safe, have been performed in humans (Cook et al., 1992; Korpinen et al., 1993; Graham et al., 1994; Korpinen and Partanen, 1994). In this study, hiPSCMs were subjected to short-term exposure to a $50 \mathrm{~Hz}$ $\mathrm{MF}$ at $400 \mathrm{mT}$, and the results suggested that under these conditions, MF exposure does not affect the electrical activity of hiPS-CMs. The flux intensity of MF applied to cardiomyocytes in this study is higher than that in previous reports. Simple calculations based on the positions of the electrodes on an MEA probe indicated that the maximum strength of the induced electric fields to which the hiPS-CMs were exposed in this experiment was approximately $380 \mathrm{mV} / \mathrm{m}$, which is approximately 4-fold higher than the basis for restriction $(90 \mathrm{mV} / \mathrm{m}$ at $50 \mathrm{~Hz})$ specified in the ICNIRP guidelines (International Commission on Non-Ionizing Radiation Protection, 2010). In the IEEE standard, the median in situ induced electric field strength threshold for cardiac excitation is set at $12 \mathrm{~V} / \mathrm{m}$ at $50 \mathrm{~Hz}$ (The Institute of Electrical and Electronics Engineers, 2002). The data reported here are valuable for experimen- tally verifying the safety and reliability of these guidelines and standard values, which are based on theoretical models. It is expected that future experiments using hiPS-CMs and stronger MF exposure would give us more reliable threshold values of the effects of $50 \mathrm{~Hz} \mathrm{MF}$ on human CMs.

The effect of low-frequency EMFs on intracellular calcium transients of CMs has been reported previously (Sert et al., 2011; Fixler et al., 2012). Low-frequency EMFs were found to change intracellular calcium concentration levels and the frequency of calcium transients in nonhuman CMs. Based on the strong correlation between the results of intracellular calcium imaging and those of extracellular FP recordings (Fujiwara et al., 2008), MFs of $50 \mathrm{~Hz}$ at $400 \mathrm{mT}$ might not affect intracellular calcium transients in hiPS-CMs. This discrepancy might be explained by interspecies differences described above. However, it should be mentioned that we evaluated the influence of MFs on hiPS-CMs by comparing the FPs of pre- and post-exposure cells. We were not able to analyze the FPs of cells during MF exposure because of the noise generated during MF exposure. Recently, we developed a non-conductive, miniature fiber-optic imaging system (Saito et al., 2017). This system enables realtime imaging of intracellular calcium transients in electrically activated cells, such as neurons and CMs, during MF exposure without any artifacts. If real-time imaging of hiPS-CMs activities in MFs is performed with this system, previously undetectable phenomena might be able to be detected.

In conclusion, valuable knowledge about the effects of power-frequency MFs on the electrical activity of human CMs was acquired using hiPS cell technology. HiPS-CMs allowed evaluation of human responsiveness to strong MFs, which is impossible to perform using primary animal cultures, experimental animals and cells, or humans. Various types of human cells derived from iPS cells could be utilized for assessment of the health risks of various targets, including EMFs. The current international guidelines/standards for low-frequency EMF exposure deserve further investigation on many points, and experimental data on human neural responsiveness to EMFs have attracted much interest in recent years (Legros et al., 2012 , 2015). We propose that data on muscles, in particular CMs, should be similarly collected for evaluation of the risk of exposure to EMFs.

\section{ACKNOWLEDGMENTS}

The authors wish to thank Ms. Mie Amari, Mr. Takuya Takahashi and Ms. Chinami Ishiyama (Ceres 
Co. Ltd.) for their assistance with the maintenance of hiPS cells and hiPS-CMs. We would also like to thank Editage for providing editorial assistance.

Conflict of interest---- The authors declare that there is no conflict of interest.

\section{REFERENCES}

Boukens, B.J., Rivaud, M.R., Rentschler, S. and Coronel, R. (2014): Misinterpretation of the mouse ECG: 'musing the waves of Mus musculus'. J. Physiol., 592, 4613-4626.

Caspi, O., Itzhaki, I., Kehat, I., Gepstein, A., Arbel, G., Huber, I., Satin, J. and Gepstein, L. (2009): In vitro electrophysiological drug testing using human embryonic stem cell derived cardiomyocytes. Stem Cells Dev., 18, 161-172.

Cook, M.R., Graham, C., Cohen, H.D. and Gerkovich, M.M. (1992): A replication study of human exposure to 60-Hz fields: effects on neurobehavioral measures. Bioelectromagnetics, 13, 261-285.

Cornacchione, M., Pellegrini, M., Fassina, L., Mognaschi, M.E., Di Siena, S., Gimmelli, R., Ambrosino, P., Soldovieri, M.V., Taglialatela, M., Gianfrilli, D., Isidori, A.M., Lenzi, A. and Naro, F. (2016): $\beta$-Adrenergic response is counteracted by extremelylow-frequency pulsed electromagnetic fields in beating cardiomyocytes. J. Mol. Cell. Cardiol., 98, 146-158.

Denning, C., Borgdorff, V., Crutchley, J., Firth, K.S., George, V., Kalra, S., Kondrashov, A., Hoang, M.D., Mosqueira, D., Patel, A., Prodanov, L., Rajamohan, D., Skarnes, W.C., Smith, J.G. and Young, L.E. (2016): Cardiomyocytes from human pluripotent stem cells: From laboratory curiosity to industrial biomedical platform. Biochim. Biophys. Acta, 1863, 1728-1748.

Elmas, O., Comlekci, S. and Koylu, H. (2012): Effects of short-term exposure to powerline-frequency electromagnetic field on the electrical activity of the heart. Arch. Environ. Occup. Health, 67, 65-71.

Fixler, D., Yitzhaki, S., Axelrod, A., Zinman, T. and Shainberg, A. (2012): Correlation of magnetic AC field on cardiac myocyte $\mathrm{Ca}(2+)$ transients at different magnetic DC levels. Bioelectromagnetics, 33, 634-640.

Fozzard, H.A. and Schoenberg, M. (1972): Strength-duration curves in cardiac Purkinje fibres: effects of liminal length and charge distribution. J. Physiol., 226, 593-618.

Fujiwara, K., Tanaka, H., Mani, H., Nakagami, T. and Takamatsu, T. (2008): Burst emergence of intracellular Ca2+ waves evokes arrhythmogenic oscillatory depolarization via the $\mathrm{Na}+-\mathrm{Ca} 2+$ exchanger: simultaneous confocal recording of membrane potential and intracellular $\mathrm{Ca} 2+$ in the heart. Circ. Res., 103, 509-518.

Funakoshi, S., Miki, K., Takaki, T., Okubo, C., Hatani, T., Chonabayashi, K., Nishikawa, M., Takei, I., Oishi, A., Narita, M., Hoshijima, M., Kimura, T., Yamanaka, S. and Yoshida, Y. (2016): Enhanced engraftment, proliferation, and therapeutic potential in heart using optimized human iPSC-derived cardiomyocytes. Sci. Rep., 6, 19111.

Geddes, L.A., Cabler, P., Moore, A.G., Rosborough, J. and Tacker, W.A. (1973): Threshold 60-Hz current required for ventricular fibrillation in subjects of various body weights. IEEE Trans. Biomed. Eng., 20, 465-468.

Graham, C., Cook, M.R., Cohen, H.D. and Gerkovich, M.M. (1994): Dose response study of human exposure to $60 \mathrm{~Hz}$ elec- tric and magnetic fields. Bioelectromagnetics, 15, 447-463.

Halbach, M., Egert, U., Hescheler, J. and Banach, K. (2003): Estimation of action potential changes from field potential recordings in multicellular mouse cardiac myocyte cultures. Cell. Physiol. Biochem., 13, 271-284.

International Commission on Non-Ionizing Radiation Protection (2010): Guidelines for limiting exposure to time-varying electric and magnetic fields (1 Hz to $100 \mathrm{kHz}$ ). Health Phys., 99, 818836.

Kawamura, M., Miyagawa, S., Miki, K., Saito, A., Fukushima, S., Higuchi, T., Kawamura, T., Kuratani, T., Daimon, T., Shimizu, T., Okano, T. and Sawa, Y. (2012): Feasibility, safety, and therapeutic efficacy of human induced pluripotent stem cell-derived cardiomyocyte sheets in a porcine ischemic cardiomyopathy model. Circulation, 126, S29-S37.

Khan, J.M., Lyon, A.R. and Harding, S.E. (2013): The case for induced pluripotent stem cell-derived cardiomyocytes in pharmacological screening. Br. J. Pharmacol., 169, 304-317.

Korpinen, L. and Partanen, J. (1994): Influence of $50 \mathrm{~Hz}$ electric and magnetic fields on the pulse rate of human heart. Bioelectromagnetics, 15, 503-512.

Korpinen, L., Partanen, J. and Uusitalo, A. (1993): Influence of 50 $\mathrm{Hz}$ electric and magnetic fields on the human heart. Bioelectromagnetics, 14, 329-340.

Legros, A., Corbacio, M., Beuter, A., Modolo, J., Goulet, D., Prato, F.S. and Thomas, A.W. (2012): Neurophysiological and behavioral effects of a $60 \mathrm{~Hz}, 1,800 \mu \mathrm{T}$ magnetic field in humans. Eur. J. Appl. Physiol., 112, 1751-1762.

Legros, A., Modolo, J., Brown, S., Roberston, J. and Thomas, A.W. (2015): Effects of a $60 \mathrm{~Hz}$ Magnetic Field Exposure Up to 3000 muT on Human Brain Activation as Measured by Functional Magnetic Resonance Imaging. PLoS One, 10, e0132024.

Mouchawar, G.A., Geddes, L.A., Bourland, J.D. and Pearce, J.A. (1989): Ability of the Lapicque and Blair strength-duration curves to fit experimentally obtained data from the dog heart. IEEE Trans. Biomed. Eng., 36, 971-974.

Nakasono, S., Laramee, C., Saiki, H. and McLeod, K.J. (2003): Effect of power-frequency magnetic fields on genome-scale gene expression in Saccharomyces cerevisiae. Radiat. Res., 160, 25-37.

Price, P.S., Keenan, R.E. and Swartout, J.C. (2008): Characterizing interspecies uncertainty using data from studies of anti-neoplastic agents in animals and humans. Toxicol. Appl. Pharmacol., 233, 64-70.

Reilly, J.P. (1998): Applied Bioelectricity: from Electrical Stimulation to Electropathology. Springer.

Reilly, J.P. (2002): Neuroelectric mechanisms applied to low frequency electric and magnetic field exposure guidelines--part I: sinusoidal waveforms. Health Phys., 83, 341-355.

Reppel, M., Igelmund, P., Egert, U., Juchelka, F., Hescheler, J. and Drobinskaya, I. (2007): Effect of cardioactive drugs on action potential generation and propagation in embryonic stem cell-derived cardiomyocytes. Cell. Physiol. Biochem., 19, 213-224.

Saito, A., Takahashi, M., Jimbo, Y. and Nakasono, S. (2017): Nonconductive and miniature fiber-optic imaging system for realtime detection of neuronal activity in time-varying electromagnetic fields. Biosens. Bioelectron., 86, 786-793.

Sert, C., Söker, S., Deniz, M. and Nergiz, Y. (2011): Intracellular $\mathrm{Ca}(2+)$ levels in rat ventricle cells exposed to extremely low frequency magnetic field. Electromagn. Biol. Med., 30, 14-20.

Sirenko, O., Cromwell, E.F., Crittenden, C., Wignall, J.A., Wright, F.A. and Rusyn, I. (2013): Assessment of beating parameters in 
Evaluations of the human cardiac effects of magnetic fields

human induced pluripotent stem cells enables quantitative in vitro screening for cardiotoxicity. Toxicol. Appl. Pharmacol., 273, 500-507.

Takahashi, K., Tanabe, K., Ohnuki, M., Narita, M., Ichisaka, T., Tomoda, K. and Yamanaka, S. (2007): Induction of pluripotent stem cells from adult human fibroblasts by defined factors. Cell, 131, 861-872.

The Institute of Electrical and Electronics Engineers (2002): IEEE Standard for safety levels with respect to human exposure to electromagnetic fields, 0-3 kHz. IEEE Std. C95.6.

Tran, T.H., Wang, X., Browne, C., Zhang, Y., Schinke, M., Izumo, S. and Burcin, M. (2009): Wnt3a-induced mesoderm formation and cardiomyogenesis in human embryonic stem cells. Stem Cells, 27, 1869-1878.

Wei, J., Sun, J., Xu, H., Shi, L., Sun, L. and Zhang, J. (2015):
Effects of extremely low frequency electromagnetic fields on intracellular calcium transients in cardiomyocytes. Electromagn. Biol. Med., 34, 77-84.

World Health Organization (2007): Extremely low frequency fields. Environmental Health Criteria 238.

Yazawa, M., Hsueh, B., Jia, X., Pasca, A.M., Bernstein, J.A., Hallmayer, J. and Dolmetsch, R.E. (2011): Using induced pluripotent stem cells to investigate cardiac phenotypes in Timothy syndrome. Nature, 471, 230-234.

Yu, J., Vodyanik, M.A., Smuga-Otto, K., Antosiewicz-Bourget, J., Frane, J.L., Tian, S., Nie, J., Jonsdottir, G.A., Ruotti, V., Stewart, R., Slukvin, I.I. and Thomson, J.A. (2007): Induced pluripotent stem cell lines derived from human somatic cells. Science, 318, 1917-1920. 